Ann. Biol. anim. Bioch. Biophys., 1977, 17 (3 A), 373-387.

\title{
Influence d'un excès d'insuline sur la composition corporelle et les teneurs en acides aminés libres du sang, du foie et du muscle du rat en croissance soumis ou non à une restriction azotée
}

\author{
par J. GRIZARD, J. PRUGNAUD, R. PION
}

Avec la collaboration technique de G. BAYLE, Odile BERNARD et Marie-Claude VALLUY

Laboratoire d'Efude du Métabolisme Azofé, I.N.R.A.

Theix, Saint-Genès-Champanelle 63110 Beaumont

Summary. Effect of insulin excess on body composition and free amino acid levels in blood, liver and muscle of growing rats fed a control diet or subjected to nitrogen restriction.

The experiment is carried out to study the effect of insulin on nitrogen and energy utilization. The animals are fed a control diet or subjected to a restricted protein intake. Food efficiency, body composition, plasma insulin and plasma corticosterone, blood glucose and blood urea, blood, liver and muscle free amino acid levels are recorded.

Male rats of the Sprague Dawley strain weighing $88 \mathrm{~g}$ at the beginning of the experiment are fed equal meals every $4 \mathrm{~h}$. Groups $A$ and $B$ are fed a balanced diet with 11.7 p. 100 crude protein ; groups $C$ and D receive a low protein diet with 8.2 p. 100 crude protein (table 1 ). They are given nearly the same amounts of energy, mineral and vitamins as the groups $A$ and $B$, but only 73 p. 100 of the crude protein intake. At about 1 p.m. every day, animals of groups $B$ and $D$ are injected subcutaneously with insulin (the dose is progressively increased from 0.5 to 1 unit per day). Animals of groups $A$ and $C$ are sham-injected with saline. When the subjects of one group have a mean body weight of $160 \mathrm{~g}$, they are all slaughtered at 3 p.m., i.e. 1 hour after a meal.

The results show that reducing the dietary protein supply results in a decrease in growth rate and energy efficiency. It increases body fat deposition. Insulin injection does not improve growth rate and nitrogen retention. There is a small increase of body fat deposition after insulin injection (table 2 and 3 ).

Reducing the dietary protein supply decreases blood urea. It increases plasma insulin and plasma corticosterone. Insulin injection increases blood urea. It decreases plasma corticosterone. Increments of plasma insulin and blood glucose after insulin injection are more important in rats fed the control diet than in rats subjected to a restricted protein intake (table 4).

Reducing protein supply results in a decrease of most of the free amino acids in blood. Insulin injection increases some of the essential free amino acids in blood and muscle of rats fed the control diet. It decreases most of the essential free amino acids in blood of rats subjected to a restricted protein intake. Insulin injection results in an increase of some essential free amino acids in the liver. It decreases most of the nonessential free amino acids in the blood and muscle (tables 5 and 6 ). 


\section{Introduction.}

L'insuline stimule la synthèse des protéines dans le muscle (Wool, 1972 ; London, 1972) et réduit leur catabolisme (Fulks, Li et Golberg, 1975) ; dans le foie, elle réduit le catabolisme protéique et l'uréogenèse (Mortimore et Mondon, 1970). La stimulation par l'insuline du transport et de l'incorporation des acides aminés dans le muscle se traduit par une baisse des feneurs de la plupart des acides aminés indispensables libres dans le sang des animaux soumis à quelques heures de jeûne (Felig ef Wahren, 1974). Chez le rat soumis à une restriction énergétique, elle se traduit par une baisse des teneurs de la plupart des acides aminés non indispensables libres dans le sang et des teneurs de la plupart des acides aminés indispensables libres dans le muscle (Grizard et al., 1975b). Chez le veau, la stimulation de la sécrétion d'insuline à la suite de l'ingestion d'un régime riche en produits amylacés est associée à une augmentation des teneurs de la plupart des acides aminés libres dans le muscle (Patureau-Mirand et al., 1976 ; Grizard, Patureau-Mirand et Pion, 1976).

Les modifications du métabolisme azoté entraînées par la restriction azotée sont associées à des changements de l'équilibre hormonal, en particulier de l'insuline : l'insulinémie est diminuée (Trenkle, 1974), l'insulino-sécrétion provoquée par le glucose est réduite (Milner, 1972) et la stimulation par l'insuline de la synthèse protéique est abolie (Waterlow, 1968 ; Allen, Munday et Turner, 1974).

Afin de préciser dans quelle mesure la restriction azotée entraîne des modifications des effets de l'insuline sur le métabolisme azoté, nous avons comparé l'influence de l'insuline exogène sur l'utilisation de l'azote et de l'énergie, en particulier sur les teneurs en acides aminés libres dans le sang, le foie et le muscle du rat en croissance soumis ou non à une restriction azotée.

\section{Matériel ef méthodes.}

\section{Animaux d'expérience.}

32 rałs mâles de souche "Sprague Dawley » sont élevés en cages individuelles dans des locaux éclairés de $22 \mathrm{~h}$ le soir à $10 \mathrm{~h}$ le matin, maintenus à $22^{\circ} \mathrm{C}$ et de degré hygrométrique contrôlé (60 p. 100). Ils sont sevrés vers $40 \mathrm{~g}$ ef nourris d'un régime standard.

Lorsque leur poids vif est voisin de $88 \mathrm{~g}$, les animaux sont répartis en 4 lots identiques $A, B, C$ et $D$; les animaux des lots $A$ et $B$ reçoivent le régime témoin tandis que les animaux des lots $C$ et $D$ reçoivent un régime pauvre en protéines. Les animaux des lots $B$ et $D$ reçoivent des injections sous-cutanées d'insuline (insuline zinc mixte Novo lente) effectuées tous les jours, à $13 \mathrm{~h}$; la dose diluée dans $0,5 \mathrm{ml}$ de soluté physiologique est augmentée progressivement de 0,5 à $1 \mathrm{UI}$ par rat au huitième jour du traitement. Les animaux des lots $A$ ef $C$ reçoivent des injections de soluté physiologique, effectuées dans les mêmes conditions.

Lorsqu'ils ont atteint un poids moyen de $160 \mathrm{~g}$, tous les animaux qui reçoivent le même régime sont sacrifiés le même jour entre 15 ef $16 \mathrm{~h}$, par égorgement sans anesthésie. Le sang est recueilli sur héparine ; $2,5 \mathrm{ml}$ environ sont mis dans de l'éthanol $95^{\circ}$ froid pour le dosage des acides aminés, $1 \mathrm{ml}$ est mis dans de l'acide trichloracé- 
tique 2,5 p. 100 pour le dosage de l'urée ef du glucose et $1,5 \mathrm{ml}$ sont centrifugés pour la séparation du plasma. Les surrénales, le foie, les muscles des pattes postérieures sont rapidement prélevés, pesés, congelés dans l'azote liquide et conservés à $-20^{\circ} \mathrm{C}$.

\section{Régimes ef mode d'alimentation par repas contrôlés.}

Ce sont des régimes semi-synthétiques à base de farine de hareng de Norvège supplémentée en méthionine, phénylalanine et tryptophane (tabl. 1). La composition du régime pauvre en protéines est telle que les animaux qui le consomment et dont l'ingestion est voisine de celle des animaux témoins, ingèrent approximativement autant de vitamines, de minéraux et d'énergie que ces derniers, mais seulement 73 p. 100 des protéines.

TABLEAU 1

Composition des régimes

( $\mathrm{g}$ par $\mathrm{kg}$ de matière sèche)

\begin{tabular}{|c|c|c|}
\hline & Régime témoin & $\begin{array}{l}\text { Régime pauvre } \\
\text { en protéines }\end{array}$ \\
\hline 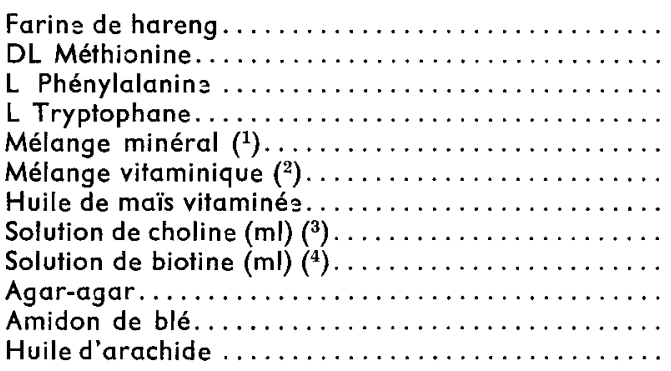 & $\begin{array}{r}146,1 \\
1,30 \\
1,01 \\
0,47 \\
40,0 \\
20,0 \\
20,0 \\
5,0 \\
5,0 \\
35,0 \\
684,5 \\
51,6\end{array}$ & $\begin{array}{r}102,2 \\
0,91 \\
0,70 \\
0,32 \\
40,0 \\
20,0 \\
20,0 \\
5,0 \\
5,0 \\
30,0 \\
727,9 \\
58,0\end{array}$ \\
\hline 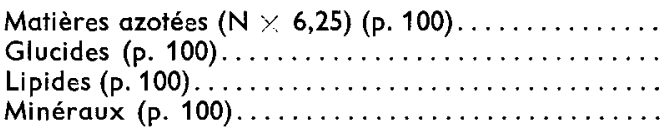 & $\begin{array}{r}11,7 \\
71,4 \\
8,0 \\
5,3\end{array}$ & $\begin{array}{c}8,24 \\
75,5 \\
8,4 \\
4,9\end{array}$ \\
\hline Energie brute (kcal par kg de matière sèche) ....... & 4333 & 4333 \\
\hline
\end{tabular}

(1) Mélange minéral utilisé par Grizard et al. (1975b).

(2) Mélange vitaminique utilisé par Pawlak ef Pion (1968a, b et c).

( $\left.{ }^{3}\right)$ Solution contenant $40 \mathrm{mg}$ par I.

(4) Solution contenant $200 \mathrm{mg}$ par $\mathrm{I}$.

Les animaux reçoivent l'aliment en 6 repas égaux par jour, distribués à 10, 14 , $18,22,2$ et $6 \mathrm{~h}$ et rapidement consommés. Le système d'alimentation par repas contrôlés est décrit par Grizard et al. (1975b) ; il permet d'éviter les modifications de rythme alimentaire ef de consommation provoquées par la restriction azotée et l'injection d'insuline et égalise les consommations des animaux d'un même lot. Les animaux disposent d'eau à volonté. 
3. Méthodes analytiques.

- Acides aminés.

Les acides aminés libres du sang sont extraits par épuisements successifs à l'éthanol 82 p. 100 contenant 2 p. 100 de thiodiglycol pour éviter l'oxydation de la méthionine. Les extraits sont purifiés sur Amberlite IR 120. Les acides aminés sont séparés et dosés sur résine échangeuse d'ions au moyen d'un analyseur automatique.

Les acides aminés libres du foie et du muscle sont dosés selon la même technique que les acides aminés libres du sang, après extraction par épuisements successifs à l'acide trichloracétique 10 p. 100 et purification sur Dowex II. L'éluat purifié est traité par la soude et le sulfate de sodium pour transformer la cystéine en cystine et éliminer l'interférence du glutathion lors de la chromatographie.

- Glucose ef urée.

Le glucose et l'urée sont dosés dans les échantillons de sang déprotéinisés à l'acide trichloracétique 2,5 p. 100 , respectivement selon les méthodes à la glucoseoxydase et la diacétyl monoxime au moyen d'une chaîne d'analyse Fontenille.

- Insuline ef corticostérone.

L'insuline est dosée par radioimmunologie selon la technique du double anticorps au moyen de la trousse de dosage in vitro CEA-IRE-SORIN INSIK 1 ; l'insuline standard est l'insuline humaine. Les dosages soni effectués d'une part dans les plasmas individuels et d'autre part dans 6 dilutions différentes des plasmas groupés par lot. Les régressions de la radioactivité du complexe insuline-anticorps anti-insuline en fonction de la concentration en insuline standard et de la radioactivité du complexe insuline-anticorps anti-insuline en fonction du facteur de dilution des plasmas, permettent de vérifier la validité des dosages ef d'estimer la valeur de l'insulinémie (tabl. 4). La corticostérone est dosée par radiocompétition selon la méthode de Murphy (1967).

- Détermination de la composition corporelle.

Les méthodes utilisées sont décrites par Grizard et al. (1975b).

\section{Méthodes statistiques.}

Les méthodes utilisées pour comparer les moyennes (analyse de variance) ou les régressions (analyse de covariance) sont celles décrites par Snedecor ef Cochran (1971).

\section{Résultats.}

1. Croissance ef efficacité alimentaire apparente des régimes (tabl. 2).

La matière sèche ingérée par jour est sensiblement plus grande chez les rats soumis à la restriction azotée que chez ceux qui reçoivent le régime témoin puisque la période pendant laquelle la consommation des animaux est élevée, est plus longue chez les rats soumis à la restriction azotée. 
La restriction azotée entraîne une nette diminution de la croissance (27 p. 100) ; l'indice de consommation augmente de 42 p. 100, le coefficient d'efficacité énergétique (CEE) diminue de 29 p. 100 ef le coefficient d'efficacité protéique (CEP) ne change pas. Quel que soit le régime consommé par les animaux, le traitement par l'insuline ne modifie ni la croissance, ni l'indice de consommation, ni les coefficients d'efficacité protéique et énergétique.

\section{TABLEAU 2}

Consommation, croissance ef efficacité alimentaire

( \pm erreur standard)

\begin{tabular}{|c|c|c|c|c|c|c|c|c|}
\hline Lots & & A & & B & & C & & D \\
\hline Régime & \multicolumn{4}{|c|}{ Témoin } & \multicolumn{4}{|c|}{ Pauvre en protéines } \\
\hline Traitement par l'insuline & & - & & + & & - & & + \\
\hline 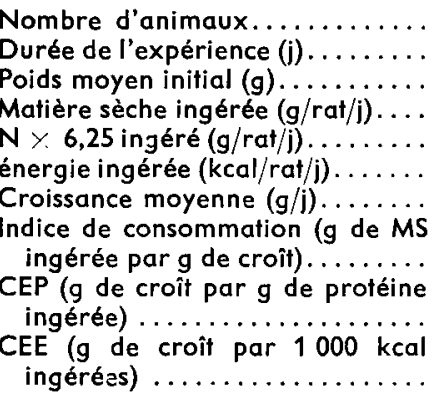 & $\begin{array}{c}88,5 \\
15,73 \\
1,840 \\
68,2 \\
5,78 \\
\\
2,73 \\
\\
3,14 \\
\\
84,8\end{array}$ & $\begin{array}{l}12 \\
12 \\
\pm 1,3 \\
\pm 0,11 \\
\pm 0,012^{\mathrm{cd}} \\
\pm 0,5 \\
\pm 0,12^{\mathrm{cd}} \\
\pm 0,06 \mathrm{~cd} \\
\pm 0,06 \\
\pm 1,7 \mathrm{~cd}\end{array}$ & $\begin{array}{c}88,0 \\
15,22 \\
1,781 \\
65,9 \\
5,79 \\
2,63 \\
\\
3,28 \\
\\
88,5\end{array}$ & $\begin{array}{l}8 \\
12 \\
\pm 1,1 \\
\pm 0,48 \mathrm{~cd} \\
\pm 0,056 \mathrm{~cd} \\
\pm 2,1 \mathrm{~cd} \\
\pm 0,08 \mathrm{~cd} \\
\pm 0,08 \mathrm{~cd} \\
\pm 0,11 \\
\pm 3,1 \mathrm{~cd}\end{array}$ & $\begin{array}{l}87,4 \\
16,19 \\
1,334 \\
70,1 \\
4,21 \\
3,87 \\
\\
3,15\end{array}$ & $\begin{array}{l}8 \\
18 \\
\pm 1,4 \\
\pm 0,28 \mathrm{~b} \\
\pm 0,023^{\mathrm{ab}} \\
\pm 1,2^{\mathrm{b}} \\
\pm 0,18^{\mathrm{ab}} \\
\pm 0,11^{\mathrm{ab}} \\
\pm 0,08 \\
\pm 1,6^{\mathrm{ab}}\end{array}$ & $\begin{array}{c}88,0 \\
16,35 \\
1,347 \\
70,8 \\
4,33 \\
3,81 \\
3,20 \\
60,9\end{array}$ & $\begin{array}{l}8 \\
18 \\
\pm 1,1 \\
\pm 0,29 \mathrm{~b} \\
\pm 0,024 \mathrm{ab} \\
\pm 1,3 \mathrm{~b} \\
\pm 0,19 \mathrm{ab} \\
\pm 0,12^{\mathrm{ab}} \\
\pm 0,09 \\
\pm 1,7^{\mathrm{ab}}\end{array}$ \\
\hline
\end{tabular}

$a, b, c, d$ : moyenne significativement différente $(P<0,050)$ des moyennes respectives obtenues pour les lots correspondants A, B, C et D.

2. Composition du corps des animaux au moment du sacrifice (tabl. 3).

La restriction azotée entraîne une nette augmentation de la teneur en lipides (59 p. 100) et en cendres (29 p. 100), une faible baisse de la teneur en eau et en protéines (respectivement 7 ef 5 p. 100) et une réduction significative du poids du foie el des surrénales (respectivement 6 et 10 p. 100). Quel que soit le régime consommé par les animaux, le traitement par l'insuline entraîne un accroissement notable de la teneur en lipides et une augmentation significative du poids des surrénales (environ 10 p. 100).

3. Glycémie, urémie, insulinémie et corticostéronémie (łabl. 4).

En ce qui concerne le dosage de l'insuline, le coefficient de corrélation associé aux régressions $\log$ it $(B / B o)=M(\ln X)+1$ a une valeur très élevée, les pentes $M$ de ces régressions sont sensiblement les mêmes et les valeurs de l'insulinémie obtenues à la 
suite des dosages effectués dans les plasmas groupés par lot et dilués, sont égales aux valeurs de l'insulinémie obtenues à la suite des dosages effectués dans les plasmas individuels.

TABLEAU 3

Composition corporelle des animaux au moment du socrifice ( \pm erreur standard)

\begin{tabular}{|c|c|c|c|c|}
\hline Lots & A & B & $\mathrm{C}$ & $\mathrm{D}$ \\
\hline Régime & \multicolumn{2}{|c|}{ Témoin } & \multicolumn{2}{|c|}{ Pauvre en protéines } \\
\hline Traitement par l'insuline & - & + & - & + \\
\hline 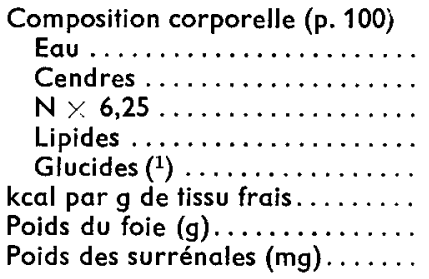 & $\begin{array}{c}66,3 \\
3,4 \\
17,3 \\
10,5 \\
2,5 \\
2,056 \\
7,27 \pm 0,11 \mathrm{~cd} \\
18,97 \pm 0,42^{\mathrm{bc}}\end{array}$ & $\begin{array}{c}65,1 \\
3,5 \\
17,2 \\
11,6 \\
2,6 \\
2,152 \\
7,11 \pm 0,11 \\
21,22 \pm 0,73 \text { acd }\end{array}$ & $\begin{array}{c}61,5 \\
4,4 \\
16,5 \\
16,7 \\
0,9 \\
2,513 \\
6,86 \pm 0,08^{a} \\
17,00 \pm 0,48^{\mathrm{abd}}\end{array}$ & $\begin{array}{c}61,0 \\
4,7 \\
16,6 \\
17,8 \\
0,0 \\
2,589 \\
6,78 \pm 0,19 a \\
18,57 \pm 0,41 b c\end{array}$ \\
\hline
\end{tabular}

$a, b, c, d$ : moyenne significativement différente $(P<0,050)$ des moyennes respectives obtenues pour les lots correspondants A, B, C et D.

(1) estimés à partir du bilan des masses ef de l'énergie.

La restriction azotée entraîne une réduction de l'urémie (61 p. 100) et une augmentation de l'insulinémie (19 p. 100) et de la corticostéronémie (46 p. 100). L'injection d'insuline a pour conséquence une augmentation de l'insulinémie (47 et 94 p. 100 respectivement chez les rats soumis à la restriction azotée et chez les rats qui reçoivent le régime témoin), un accroissement de l'urémie (104 et 57 p. 100 respectivement chez les rats soumis à la restriction azotée et chez les rats qui reçoivent le régime témoin), une augmentation de la glycémie des rats qui reçoivent le régime témoin (37 p. 100) et une baisse considérable de la corticostéronémie (environ 87 p. 100).

\section{Acides aminés (łabl. 5 et 6 ).}

a) Teneurs en acides aminés libres du sang.

- Acides aminés indispensables et semi-indispensables.

La restriction azotée entraîne une diminution importante des teneurs en histidine (75 p. 100) lysine, et valine (environ 50 p. 100) et une baisse modérée des teneurs en thréonine, isoleucine, leucine, phénylalanine et arginine (entre 19 et 27 p. 100). Les parts de l'histidine, de la lysine et de la valine dans la somme des teneurs en acides aminés libres indispensables et semi-indispensables diminuent, alors que celles de tous les autres acides aminés libres indispensables et semi-indispensables augmentent. 


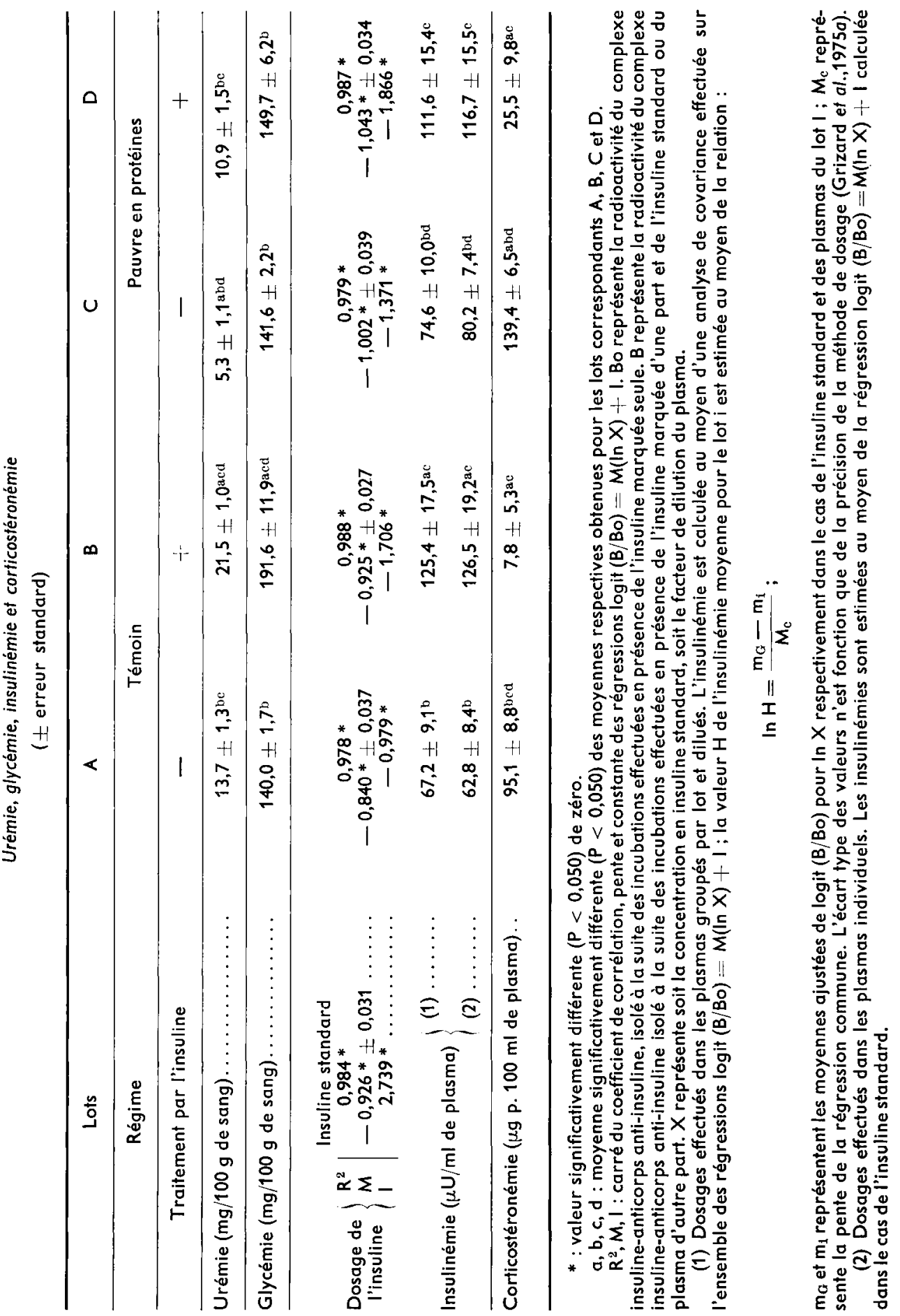


Le traitement par l'insuline des animaux qui reçoivent le régime témoin entraîne une nette diminution de la teneur en valine (32 p. 100), une augmentation modérée des teneurs en leucine, phénylalanine et thréonine (entre 13 et 23 p.100) et une augmentation importante de la teneur en tyrosine $(60$ p. 100). Le traitement par l'insuline des animaux qui reçoivent le régime pauvre en protéines provoque une très nette réducfion des teneurs en arginine, méthionine et valine (environ 40 p. 100), une baisse modérée des teneurs en lysine, leucine et thréonine (entre 18 et 26 p. 100) et une augmentation des teneurs en isoleucine, tyrosine ef phénylalanine (respectivement 50,40 et 25 p. 100). La part de l'histidine dans la somme des teneurs en acides aminés libres indispensables et semi-indispensables augmente alors que celles de la lysine, de la leucine ef de la thréonine ne changent pas.

TABLEAU 5

Teneurs en acides aminés libres du sang, du foie et du muscle (mg par $100 \mathrm{~g}$ de tissu frais)

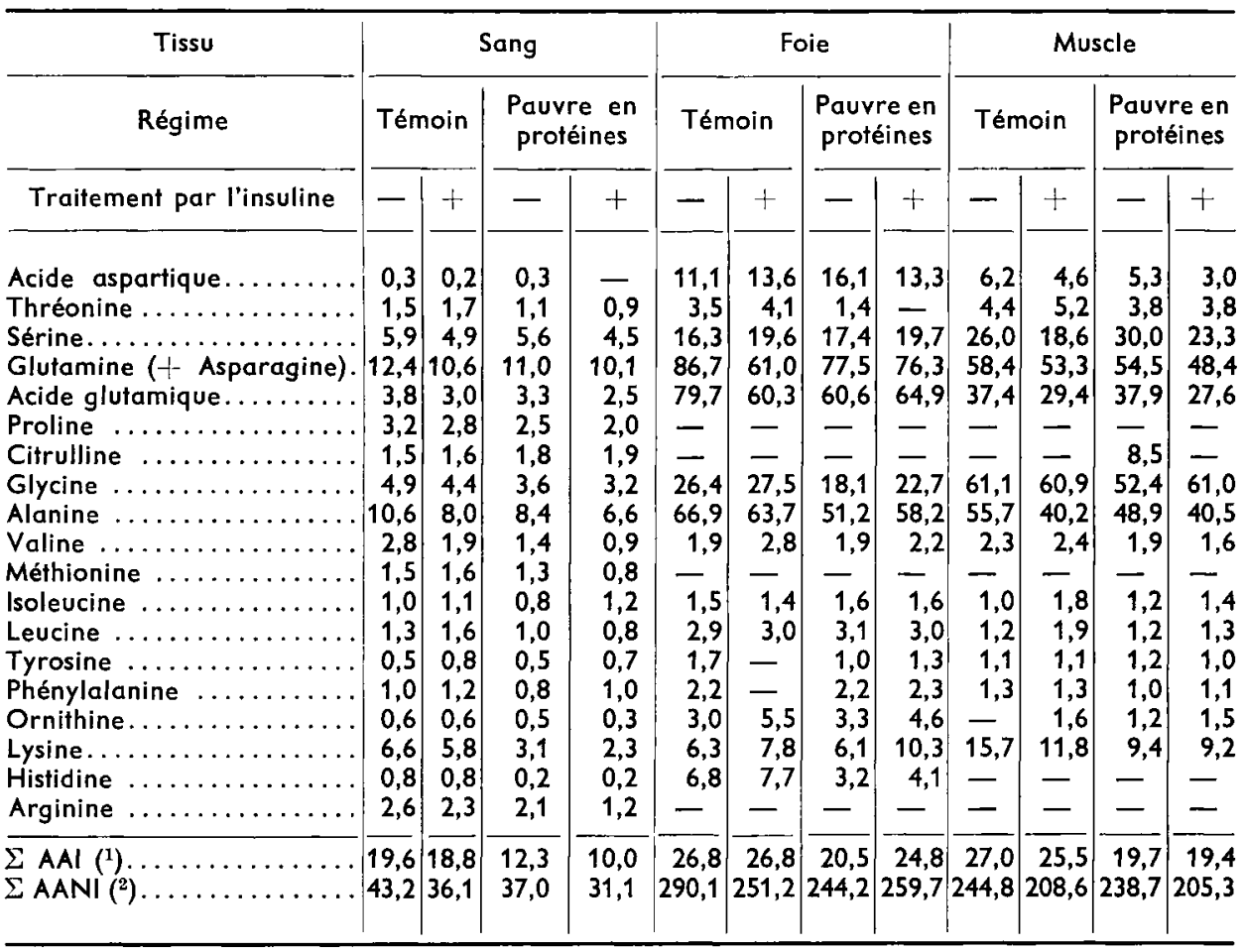

(1) Somme des teneurs en acides aminés indispensables et semi-indispensables.

(2) Somme des teneurs en acides aminés non indispensables.

- Acides aminés non indispensables

La restriction azotée entraîne une baisse modérée des teneurs en glycine, proline, alanine, ornithine et acide glutamique (entre 13 et 26 p. 100) et une augmentation de 
la teneur en citrulline (19 p. 100). Le traitement par l'insuline des animaux qui reçoivent le régime témoin entraîne une nette diminution de la teneur en acide aspartique ( 33 p. 100) et une baisse modérée des teneurs en alanine, acide glutamique, sérine ef glutamine plus asparagine (entre 12 et 24 p. 100). Le traitement par l'insuline des animaux qui reçoivent le régime pauvre en protéines entraîne une forte diminution des teneurs en acide aspartique et ornithine (respectivement 100 et 40 p. 100) et une baisse modérée des teneurs en acide glutamique, alanine, proline et sérine.

\section{TABLEAU 6}

Teneurs en acides aminés libres indispensables et semi-indispensables (p. 100 de leur somme)

\begin{tabular}{|c|c|c|c|c|c|c|c|c|c|c|c|c|}
\hline \multirow{3}{*}{$\begin{array}{c}\text { Tissu } \\
\text { Régime } \\
\text { Traitement par l'insuline }\end{array}$} & \multicolumn{4}{|c|}{ Sang } & \multicolumn{4}{|c|}{ Foie } & \multicolumn{4}{|c|}{ Muscle } \\
\hline & \multicolumn{2}{|c|}{ Témoin } & \multicolumn{2}{|c|}{$\begin{array}{l}\text { Pauvre en } \\
\text { protéines }\end{array}$} & \multicolumn{2}{|c|}{ Témoin } & \multicolumn{2}{|c|}{$\begin{array}{l}\text { Pauvre en } \\
\text { protéines }\end{array}$} & \multicolumn{2}{|c|}{ Témoin } & \multicolumn{2}{|c|}{$\begin{array}{c}\text { Pauvre en } \\
\text { protéines }\end{array}$} \\
\hline & -1 & + & - & + & - & + & - & + & - & + & - & + \\
\hline Thréoni & 7,6 & 9,0 & 8,9 & 9,0 & 13,0 & 15,3 & 6,8 & - & 16,3 & 20,4 & 19,3 & 19,6 \\
\hline Valir & 14,3 & 10,1 & 11,4 & 9,0 & 7,1 & 10,4 & 9,3 & 8,8 & 8,5 & 9,4 & 9,6 & 8,2 \\
\hline Méthionine & 7,6 & 8,5 & 10,6 & 8,0 & - & - & - & - & - & - & - & - \\
\hline Isoleucin & 5,1 & 5,8 & 6,5 & 12,0 & 5,6 & 5,2 & 7,8 & 6,4 & 3,7 & 7,0 & 6,1 & 7,2 \\
\hline Leucine & 6,6 & 8,5 & 8,1 & 8,0 & 10,8 & 11,2 & 15,1 & 12,1 & 4,4 & 7,4 & 6,1 & 6,7 \\
\hline Tyrosine & 2,5 & 4,2 & 4,1 & 7,0 & 6,3 & - & 4,9 & 5,2 & 4,1 & 4,3 & 6,1 & 5,1 \\
\hline Phénylalanine & 5,1 & 6,4 & 6,5 & 10,0 & 8,2 & - & 10,7 & 9,3 & 4,8 & 5,1 & 5,1 & 5,7 \\
\hline Lysine. . & 33,7 & 30,8 & 25,2 & 23,0 & 23,5 & 29,1 & 29,7 & 41,5 & 58,1 & 46,3 & 47,7 & 47,4 \\
\hline Histidine & 4,1 & 4,2 & 1,6 & 2,0 & 25,4 & 28,7 & 15,6 & 16,5 & - & - & - & - \\
\hline Arginine & 13,3 & 12,2 & 17,1 & 12,0 & - & - & - & - & - & - & - & - \\
\hline
\end{tabular}

b) Teneurs en acides aminés libres du foie.

- Acides aminés indispensables et semi-indispensables.

La restriction azotée entraîne une diminution importante des teneurs en thréonine, tyrosine et histidine. Les parts des acides aminés à chaîne ramifiée (leucine, isoleucine et valine), de la phénylalanine et de la lysine dans la somme des teneurs en acides aminés libres indispensables et semi-indispensables augmentent très nettement.

Le traitement par l'insuline des animaux qui reçoivent le régime témoin entraîne une baisse considérable des teneurs en tyrosine et phénylalanine (100 p. 100), un accroissement modéré des teneurs en lysine, thréonine et histidine (entre 13 et 24 p. 100) et une forte augmentation de la teneur en valine (47 p. 100). Le traitement par l'insuline des animaux qui reçoivent le régime pauvre en protéines entraîne une réduction considérable de la teneur en thréonine (100 p. 100), une légère augmentation de la teneur en valine (16 p. 100), un net accroissement des teneurs en tyrosine et histidine (environ 30 p. 100) et une augmentation importante de la teneur en lysine (69 p. 100). Les parts de la leucine, de l'isoleucine et de la phénylalanine dans la somme des teneurs en acides aminés libres indispensables et semi-indispensables diminuent légèrement alors que celles de la tyrosine, de l'histidine et de la valine restent constantes 
- Acides aminés non indispensables.

La restriction azotée provoque une baisse notable des teneurs en glycine, acide glutamique et alanine (entre 23 et 31 p. 100) et une forte augmentation de la teneur en acide aspartique (45 p. 100). Le traitement par l'insuline des animaux qui reçoivent le régime témoin entraîne une baisse des teneurs en glutamine plus asparagine et acide glutamique (respectivement 30 et 24 p. 100), un accroissement modéré des teneurs en acide aspartique et sérine (environ 21 p. 100) ef une forte augmentation de la teneur en ornithine (83 p. 100). Le traitement par l'insuline des animaux qui reçoivent le régime pauvre en protéines provoque une baisse modérée de la teneur en acide aspartique (17 p. 100), un accroissement modéré des teneurs en glycine, alanine tł sérine (entre 13 et 25 p. 100) et une très nette augmentation de la teneur en ornithine ( 39 p. 100).

\section{c) Teneurs en acides aminés libres du muscle.}

- Acides aminés indispensables et semi-indispensables.

La restriction azotée provoque une forte diminution de la teneur en lysine (40 p. 100), une baisse modérée des teneurs en phénylalanine, valine $\epsilon \dagger$ thréonine (entre 14 et 23 p. 100) et un accroissement notable de la teneur en isoleucine ( 20 p. 100). Les parts de tous les acides aminés dans la somme des teneurs en acides aminés libres indispensables et semi-indispensables augmentent, à l'exception de la part de la lysine qui diminue sensiblement. Le traitement par l'insuline des animaux qui reçoivent le régime témoin entraîne une nette baisse de la teneur en lysine (25 p. 100), un accroissement modéré de la teneur en thréonine (18 p. 100) ef une très forte augmentation des t€neurs en isoleucine et leucine (respectivement 58 et 80 p. 100). Le traitement par l'insuline des animaux qui reçoivent le régime pauvre en protéines provoque une baisse modérée des teneurs en tyrosine et valine (environ 17 p. 100) et un accroissement modéré de la teneur en isoleucine (17 p. 100).

- Acides aminés non indispensables.

La restriction azotée provoque une légère baisse des teneurs en acide aspartique, glycine et alanine (entre 12 et 14 p. 100), un léger accroissement de la teneur en sérine (15 p. 100) et une accumulation de la citrulline et de l'ornithine. Le traitement par l'insuline des animaux qui reçoivent le régime témoin entraîne une baisse notable des teneurs en sérine, alanine, acide aspartique et acide glutamique (entre 21 et 28 p. 100) ef une accumulation de l'ornithine. Le traitement par l'insuline des animaux qui reçoivent le régime pauvre en protéines entraîne une forte réduction de la teneur en citrulline et acide aspartique, une baisse modérée des teneurs en acide glutamique, sérine et alanine (entre 17 et 27 p. 100) ef un accroissement modéré des teneurs en ornithine et glycine (respectivement 25 et 16 p. 100).

\section{Discussion.}

1. Validité du dosage de l'insuline.

Le parallélisme entre les droiłes qui représentent la radioactivité du complexe insuline-anticorps anti-insuline en fonction de la dilution des plasmas et celle qui représente la radioactivité du complexe insuline-anticorps anti-insuline en fonction de la 
concentration en insuline standard, montre que les substances dosées ont la même affinité pour les anticorps anti-insuline que l'insuline humaine; ceci signifie que les $\alpha$ globulines et l'albumine plasmatiques n'interfèrent probablement pas au cours des dosages (Felix, Sutter-Dub et Legrele, 1975).

\section{Insulinémie.}

La restriction azotée n'entraîne pas de baisse de l'insulinémie, contrairement à ce qui est habituellement constaté chez les animaux nourris ad libifum (Trenkle, 1974); ceci peut s'expliquer en partie par le fait que le mode d'alimentation a pour conséquence des valeurs faibles de l'insulinémie chez les rats qui reçoivent le régime témoin.

L'accroissement de l'insulinémie des rats à la suite de l'injection d'insuline est beaucoup plus forte chez les rats qui reçoivent le régime témoin que chez ceux qui reçoivent le régime pauvre en protéines. Ceci est dû soit à une augmentation de la sécrétion, soit à une réduction du catabolisme de l'insuline chez les rats qui reçoivent le régime témoin, provoquée par une réduction de la sensibilité des tissus à l'insuline (Salganik et al., 1974).

\section{Fonctionnement des corticosurrénales.}

La restriction azotée entraîne une augmentation de la corticostéronémie malgré une réduction du poids des surrénales. Ceci peut résulter du fait que la réduction du catabolisme de l'hormone dans le foie (Anon., 1974) est plus grande que la réduction de la sécrétion de l'hormone par la corticosurrénale (Munro, 1964).

Le traitement par l'insuline entraîne une forte baisse de la corticostéronémie contrairement à l'augmentation sensible de la glucocorticoïdémie constatée chez diverses espèces animales à la suite de l'injection d'une seule dose d'insuline (Donald, Salisbury Murphy ef Nabarro, 1968 ; Zimmermann-Telschow et al., 1969) et chez le rat à la suite d'injections répétées de faibles doses d'insuline (Schulke et al., 1973). Ceci peut s'expliquer par une réduction de la sécrétion de corticostérone associée à l'absence d'hypoglycémie puisque Zukoski (1966) a montré que la décharge de glucocorticoïdes provoquée par l'injection d'insuline ne résulte que d'une baisse de la glycémie.

\section{Glycémie.}

L'accroissement de la glycémie, provoqué par le traitement par l'insuline des rats qui reçoivent le régime témoin est en accord avec les constatations de Salganik ef al. (1974) effectuées chez le rat en croissance. Ces auteurs ont montré que l'injection journalière, pendant plus de 10 jours, de fortes doses d'insuline (2 UI par rat, administrées par voie sous-cutanée) provoque un accroissement de la glycémie dû à une réduction de l'utilisation du glucose par le foie à la suite d'une baisse de l'activité des enzymes de la glycolyse (hexokinase, pyruvate kinase) associée à une réduction de la sensibilité à l'insuline.

\section{Utilisation de l'azote et de l'énergie.}

Le fait que la quantité de protéines fixée diminue sensiblement et que la quantité de protéines ingérées ne change pas, signifie que la restriction azotée entraîne une légère baisse du coefficient de rétention azotée. L'augmentation modérée de l'énergie 
fixée associée à la forte augmentation de l'énergie ingérée montre que la restriction azotée entraîne une réduction du coefficient de rétention énergétique.

Aussi bien chez les rats qui consomment le régime témoin que chez ceux qui reçoivent le régime pauvre en protéines, le traitement par l'insuline n'améliore pas la rétention azotée, contrairement à ce qui a été constaté chez les animaux nourris ad libitum (Snipes, 1968) et les rats soumis à une restriction énergétique (Grizard et al., 1975b) ou recevant séparément les protéines et l'énergie (Henry, 1976). Chez les animaux témoins, ce résultat est en contradiction avec le fait que l'insuline accroît la synthèse at réduit le catabolisme protéique musculaire (Wool, 1972 ; London, 1972 ; Fulks, Li et Golberg, 1975) ; chez les animaux qui reçoivent le régime pauvre en protéines, il est en accord avec les constatations de Waterlow (1968) et Allen, Munday et Turner (1974) selon lesquelles l'insuline ne stimule pas la synthèse des protéines chez le rat ef le lapin soumis à une restriction azotée. L'augmentation de l'adiposité du corps des animaux, à la suite du traitement par l'insuline a souvent été constatée (Snipes, 1968).

6. Teneurs en acides aminés libres du sang, du foie et du muscle.

a) Influence de la restriction azotée.

La diminution des teneurs de la plupart des acides aminés libres indispensables et semi-indispensables dans le sang et des teneurs en lysine, phénylalanine, valine et thréonine libres dans le muscle est due à la réduction de l'apport de ces derniers à la suite de la consommation du régime pauvre en protéines (Young ef al., 1973). La teneur en lysine libre dans le muscle est nettement plus élevée que la teneur trouvée par Pawlak et Pion (1968a) chez des rats qui consommaient un régime contenant la même quantité de lysine que le régime pauvre en protéines et approximativement les mêmes quantités des autres acides aminés que le régime témoin ; ceci est en accord avec les constatations de Pawlak et Pion (1968c) et résulte vraisemblablement du fait que la lysine est moins utilisée puisque la vitesse de croissance des animaux est nettement plus faible. Dans le sang et le muscle, le fait que la baisse de la teneur en lysine libre est nettement plus forte que la baisse de la teneur en thréonine libre est en accord avec les courbes qui représentent les teneurs en lysine et thréonine libres sanguines et musculaires en fonction des teneurs en lysine et thréonine dans le régime (Pawlak et Pion $1968 a$ et $b$ ) ; pour des teneurs en lysine et thréonine libres correspondant à celles trouvées chez les animaux qui consomment le régime témoin, une réduction de $30 \mathrm{p} .100$ de la teneur de ces acides aminés dans le régime se traduit par une baisse beaucoup plus forte des teneurs en lysine que des teneurs en thréonine.

La baisse modérée des teneurs de la plupart des acides aminés libres non indispensables dans le sang, des teneurs en glycine, acide glutamique et alanine libres dans le foie, des teneurs en acide aspartique, glycine ef alaline libres dans le muscle, peut s'expliquer aussi bien par la réduction de l'apport de ces derniers à la suite de la consommation du régime pauvre en protéines que par un ralentissement de la synthèse des acides aminés non indispensables à partir des acides aminés indispensables.

L'accroissement des teneurs en citrulline libre dans le sang ef le muscle est probablement dû à une augmentation de la libération de citrulline par le foie à la suite 
d'une baisse de l'utilisation de cet acide aminé pour l'uréogenèse hépatique ; une relation entre les teneurs en citrulline libre sanguine et musculaire et l'uréogenèse a déjà été constatée (Grizard ef al., 1975b ; Grizard, Prugnaud et Pion, 1974).

b) Influence de l'insuline.

Chez les animaux qui reçoivent le régime témoin, l'accroissement des teneurs en tyrosine, leucine, phénylalanine, thréonine et isoleucine libres dans le sang et des teneurs en isoleucine, leucine ef thréonine libres dans le muscle, sans modification de la rétention azotée, peut s'expliquer par un ralentissement du catabolisme de ces acides aminés.

Chez les animaux qui ingèrent le régime pauvre en protéines, la baisse des teneurs sanguines de la plupart des acides aminés libres indispensables (méthionine, valine, arginine, lysine, leucine et thréonine) est vraisemblablement due en partie à l'accroissement du passage des acides aminés du sang vers le muscle, puisque l'insuline stimule le transport des acides aminés dans le muscle et leur incorporation dans les protéines (Wool, 1972 ; London, 1972). Le fait que ni la rétention azotée, ni les teneurs musculaires des acides aminés libres indispensables n'augmentent, montre que le catabolisme de ces acides aminés est accru, contrairement à ce qui est observé chez les rats qui reçoivent le régime témoin.

Quel que soit le régime consommé par les animaux, l'accroissement des teneurs de certains acides aminés indispensables libres dans le foie (valine, lysine, thréonine histidine), peut s'expliquer aussi bien par un ralentissement de l'anabolisme protéique hépatique que par un accroissement du passage des acides aminés du sang vers le foie.

La baisse des teneurs de la plupart des acides aminés non indispensables libres dans le sang et muscle, associée à la forte augmentation de l'urémie et de la glycémie chez les rats nourris avec le régime témoin, est vraisemblablement due à un accroissement du catabolisme des acides aminés non indispensables et à une stimulation de la synthèse du glucose à partir de ces derniers. Ceci est en contradiction avec le fait que l'insuline diminue la néoglucogenèse (Parilla, Jimenez et Ayuso-Parilla, 1976) et peut résulter soit d'une baisse de la sensibilité des tissus à l'insuline (Salganik, 1974), soit d'une restriction énergétique (Grizard et al., 1975b) liée à l'absence d'augmentation de la consommation des animaux à la suite du traitement hormonal.

\section{Conclusion.}

Aussi bien chez les rats qui reçoivent le régime témoin que chez ceux qui reçoivent le régime pauvre en protéines, le traitement chronique par une forte dose d'insuline n'améliore pas la rétention azotée ef provoque un accroissement du catabolisme des acides aminés non indispensables. Chez les rałs qui reçoivent le régime lémoin, le catabolisme des acides aminés indispensables semble diminué ; en revanche, chez les rats qui reçoivent le régime pauvre en protéines le transport dans le muscle et le catabolisme des acides aminés indispensables semble accru. Le traitement hormonal induit sans doute une réduction de la sensibilité des tissus à l'insuline, moins forte chez les rats qui reçoivent le régime pauvre en protéines que chez ceux qui reçoivent le régime témoin et qui se traduit en particulier par un accroissement de la glycémie et de l'insulinémie. 


\section{Références}

ALLEN K. A., MUNDAY K. A., TURNER M. R., 1974. Effects of age and postnatal diet on the protein synthetic actions of insulin and growth hormone. Proc. Nutr. Soc., 33, 113A-114A.

Anonyme, 1974. Adrenal regulation of protein synthesis in protein malnutrition. Nutr. Rev., 32, 51-52.

DONALD R. A., SALISBURY MURPHY S., NABARRO J. D. N., 1968. The plasma corticotrophin response to insulin hypoglycemia, lysine-vasopressin and metyrapone in pigs. Endocrinology, 41, 509-518.

FELIG P., WAHREN J., 1974. Protein turnover and amino acid metabolism in the regulation of gluconeogenesis. Fed. Proc., 33, 1092-1097.

FELIX J. M., SUTTER-DUB M. T., LEGRELE C., 1975. Studies on the different forms of material reacting with anti-insulin antibodies in the fetal and adult rat. Horm. Metab. Res., 7, 394-399.

FULKS R. M., LI J. B., GOLBERG A. L., 1975. Effect of insulin, glucose, and amino acids on protein turnover in rat diaphragm. J. biol. Chem., 250, 290-298.

GRIZARD G., BOUCHER D., THIEBLOT L., PITAVY J., 1975 a. Dosage biologique des gonadostimulines hypophysaires chez le rat mâle (étude statistique). C. R. Soc. Biol., 169, 581-587.

GRIZARD J., PATUREAU-MIRAND P., PION R., 1976. Utilisation d'un régime riche en produits amylacés par le veau préruminant de poids élevé. II. Influence sur l'insulinémie postprandiale. Ann. Biol. anim. Bioch. Biophys., 16, 593-601.

GRIZARD J., PRUGNAUD J., ARNAL M., PION R., 1975b. Effet de l'insuline sur la composition corporelle et les teneurs en acides aminés libres du sang, du foie et du muscle du rat en croissance soumis à une restriction énergétique. Ann. Biol. anim. Bioch. Biophys., 15, 569-582.

GRIZARD J., PRUGNAUD J., PION R., 1974. Influence de l'alimentation par repas sur la composition corporelle et les teneurs sanguines en acides aminés libres chez le Rat en croissance. C. R. Soc. Biol., 168, 738.

HENRY Y. 1976. Influence de l'insuline exogène sur l'ajustement de la consommation spontanée d'énergie chez le rat en croissance. Vile Congr. Int. Metab. énerg. Vichy.

LONDON D. R., 1972. Control by insulin of protein synthesis in muscle. Proc. Nutr. Soc., 31, 193-197.

MILNER R. D. G., 1972. Insulin secretion in human protein-calorie deficiency. Proc. Nutr. Soc., 31, 219-223.

MORTIMORE G. E., MONDON C. E., 1970. Inhibition by insulin of valine turnover in liver. J. biol. Chem., 245, 2375-2383.

MUNRO H. N., 1964. General aspects of the regulation of protein metabolism by diet and by hormones. In MUNRO H. N., ALLISON J. B., Mammalian Protein Metabolism, Vol. 1, 382-468. Acad. Press New York and London.

MURPHY B. E. P., 1967. Some studies of the protein binding of steroids and their application to the routine micro and ultramicro measurement of various steroids in body fluids by competitive protein radioassay. J. clin. Endocrin. Metab., 27, 973-990.

PARILLA R., JIMENEZ M. I., AYUSO-PARILLA M. S., 1976. Cellular redistribution of metabolites during glucagon and insulin control of gluconeogenesis in the isolated perfused rat liver. Arch. Biochem. Biophys., 174, 1-12.

PATUREAU-MIRAND P., GRIZARD J., PRUGNAUD J., PION R., 1976. Utilisation d'un aliment riche en produits amylacés par le veau préruminant de poids élevé. I. - Influence sur les teneurs en acides aminés libres du sang et du muscle. Ann. Biol. anim. Bioch. Biophys., 16, 579592.

PAWLAK M., PION R., 1968a. Influence de la supplémentation des protéines de blé par des doses croissantes de lysine sur la teneur en acides aminés libres du sang et du muscle du rat en croissance. Ann. Biol. anim. Bioch. Biophys., 8, 517-530. 
PAWLAK M., PION R., 1968b. Influence de la supplémentation des protéines du blé par des doses croissantes de thréonine sur les teneurs en acides aminés libres du sang total et du muscle du rat en croissance. C. R. Acad. Sci. Paris, Série D, 266, 1993-1995.

PAWLAK M., PION R., 1968c. Influence d'une diminution du taux protéique de la ration sur les teneurs en acides aminés libres des tissus sanguin et musculaire du rat en croissance. Ann. Biol. anim. Bioch. Biophys., 8, 457-459.

SALGANIK R. I., MERTVETSOV N. P., GORDIENKO O. E., CHESNOKOV V. N., SEMONOVA L. A., 1974. Impairment of induction of glycolytic enzymes and development of insulin resistance in rats as a result of continuous insulin treatment. Endocrinologica, 76, 319-331.

SCHULKE B., PANASENKO W. N., ROESKE H., TEGELER G., 1973. Einfluss des Insulins auf die Körperzusammensetzung, die Massezunahme und Plasmakortikosteronkonzentration von Ratten bei Ernährung mit Protein unterschiedlicher Qualität. Arch. Tierernährung, 23, 149-155.

SNEDECOR G. W., COCHRAN W. G., 1971. Méthodes statistiques. A.C.T.A. ed., Paris.

SNIPES C. A., 1968. Effects of growth hormone and insulin on amino acid and protein metabolism. Quart. Rev. Biol., 43, 127-147.

TRENKLE A., 1974. Hormonal and nutritional interrelationships and their effects on skeletal muscle. J. Anim. Sci., 38, 1142-1149.

WATERLOW J. C., 1968. Observations on the mechanism of adaptation to low protein intakes. Lancel, 7578, 1091-1097.

WOOL I. G., 1972. Insulin and the regulation of protein synthesis in muscle. Proc. Nutr. Soc., 31, 185-191.

YOUNG V. R., VILAIRE G., NEWBERNE P. M., WILSON R. B., 1973. Plasma insulin and amino acid concentrations in rats given and adequate or low protein diet. J. Nutr., 103, 720-729.

ZIMMERMANN-TELSCHOW H., HERBERG L., GRIES F. A., BETHGE H., ZIMMERMANN H., 1969. Über die Veränderung von Aminosäuren, Blutzucker, Glucocorticoiden, freien Fettsäuren und freiem Glycerin im Plasma nach Insulininjektion mit und ohne Infusion von Aminosäuren. Klin. Wochenschr, 47, 651-668.

ZUKOSKI C. F., 1966. Mechanism of action of insulin hypoglycemia on adrenal cortical secretion. Endocrinology, 78, 1264-1267. 